New Zealand Identities. Departures and Destinations de James H.LIU, Tim MCCREANOR, Tracey MCIINTOSH and Teresia TEAIWA (eds)

\title{
Raymond MAYER
}

\section{OpenEdition}

\section{Journals}

\section{Édition électronique}

URL : http://journals.openedition.org/jso/6453

DOI : 10.4000/jso.6453

ISSN : $1760-7256$

Éditeur

Société des océanistes

\section{Édition imprimée}

Date de publication : 15 décembre 2011

Pagination : 439-440

ISBN : 978-2-85430-037-4

ISSN : 0300-953x

\section{Référence électronique}

Raymond MAYER, «New Zealand Identities. Departures and Destinations de James H.LIu, Tim Mccreanor, Tracey мсіпттовн and Teresia tealwa (eds) », Journal de la Société des Océanistes [En ligne], 133 | 2e semestre 2011, mis en ligne le 31 décembre 2011, consulté le 24 septembre 2020. URL : http:// journals.openedition.org/jso/6453; DOI : https://doi.org/10.4000/jso.6453

Ce document a été généré automatiquement le 24 septembre 2020.

(C) Tous droits réservés 


\title{
New Zealand Identities. Departures and Destinations de James H.LIU, Tim MCCREANOR, Tracey MCIINTOSH and Teresia TEAIWA (eds)
}

\author{
Raymond MAYER
}

\section{RÉFÉRENCE}

LIU James H., MCCREANOR Tim, MCIINTOSH Tracey and Teresia TEAIWA (eds), 2005. New Zealand Identities. Departures and Destinations, Wellington, Victoria University Press, 304 p., photogr., postface, notices sur les contributeurs, index.

1 Cet ouvrage de vingt-deux contributeurs donne la mesure d'une question largement en débat en Nouvelle-Zélande, comme dans de nombreux pays, mais qui échappe ici - on veut du moins le croire - à sa fréquente instrumentalisation politique. Il s'agit de la question de l'identité nationale, que les éditeurs ont pris la précaution d'écrire au pluriel («New Zealand Identities») pour éviter le piège de l'identité uniforme qui sert parfois de pensée unique ailleurs. Les contributeurs, dont une notice biographique précise qu'ils sont tous citoyens néo-zélandais, mais d'origines diverses - de là, le soustitre « (points de) départs et (de) destinations » - ont essayé une posture d'analystes à partir de leur situation de migrants transnationaux et le résultat en a été édité par le Centre de recherches interculturelles appliquées de l'université Victoria de Wellington. Comme tel, l'ouvrage pourrait n'être qu'une des innombrables publications des actes d'un colloque. En raison du thème traité, son intérêt s'impose cependant comme une contribution originale à un débat de large envergure.

2 Parmi les 22 contributeurs, certains sont d'origine maori, écossaise, samoane, taïwanaise, etc. ce qui lance un débat multi-situé, et enraciné dans l'expérience du vécu de chacun d'eux. On a l'assurance que l'expérience vécue l'emporte sur la spéculation 
technocratique, et que l'épreuve des faits jugule toute tentative d'échappatoire ou de langue de bois. Il est assez rare qu'un ouvrage fasse ouvertement référence à l'origine "ethnique " de son (ou ses) auteur(s). D'habitude les scientifiques essaient de construire les récits de vie des autres. Ici, ils se mettent en scène eux-mêmes, pour réfléchir à la manière dont ils se conçoivent comme citoyens d'un pays qui est différent de leur pays natal ou de leur culture d'origine.

Dans leur préface, les coéditeurs veulent faire souffler «l'esprit de Waikanae », lieu du séminaire-atelier qui, en novembre 2004, a réuni les contributeurs sur le thème dont l'ouvrage est issu. Leur introduction, "Construire les identités de Nouvelle-Zélande » (pp.11-20), insuffle de fait les ventilations du thème sous trois convocations théoriques, à savoir que :

- les identités sont dynamiques et multidimensionnelles;

- elles sont socialement construites ;

- elles sont porteuses d'idéologie.

On n'en demandait pas moins. Le débat peut commencer. David Pearson le commence précisément en interrogeant les "mythes de l'État unitaire" discriminés dans l'affinement des concepts de «citoyenneté », d'«identité » et d'«appartenance » (pp. 21-37).

On considérera ensuite que Tracey McIntosh (pp. 38-51) trouve le ton juste en assignant à l'identité maori, elle-même déclinée au pluriel, trois modalités historiques («fixée, fluide, forcée ») qui empêchent qu'on ne l'enferme dans une définition uniforme et statique. Tim McCreanor (pp.52-68) poursuit le travail d'analyse dans la même perspective en envisageant symétriquement l'identité pakeha de la colonisation britannique par la déconstruction de leur discours récurrent sur les Maoris à travers les stéréotypes qui font ressortir des droits, des privilèges, des jugements de valeur figés et finalement des attestations de mutuelle ignorance.

6 Dans sa contribution sur " histoire et identité vues comme un système de contrôle et d'équilibre» (pp.69-87), James H. Liu part d'un point de vue de socio-psychologue. Faisant remarquer que d'autres facteurs que la nationalité organisent une population donnée, en pensant notamment à la religion, à la situation professionnelle, à la classe sociale ou à l'appartenance ethnique, l'auteur a la judicieuse idée de comparer, sur une base d'enquêtes auprès d'adultes des deux communautés d'origines respectivement européenne et maori, l'ordre d'importance des dix événements majeurs ayant marqué l'histoire de la Nouvelle-Zélande. Le traité de Waitangi arrive en tête auprès des deux groupes culturels, mais ne reçoit pas la même interprétation du fait que l'événement qui est classé en seconde position - «l'arrivée des Européens » sur le sol du Aotearoa est intitulé et interprété par le groupe maori comme «les guerres pour la terre». Autrement dit, on confirme ainsi qu'une représentation sociale est toujours dépendante de son énonciateur et n'est jamais une donnée en soi.

7 Le traité de Waitangi fait l'objet des analyses de Giselle Byrnes (pp. 88-103) qui s'appuie sur les actes du tribunal de Waitangi, instauré en 1975, pour interroger les notions de nation et d'identité et comprendre les critères de revendication qui sont mis en œuvre dans les requêtes concernant le retour des terres à leurs propriétaires lignagers d'avant la colonisation du XIX ${ }^{\mathrm{e}}$ siècle.

8 Le refus de l'essentialisme culturel marque la contribution de H.B. Levine (pp. 104-117) pour écrire sur les rapports de citoyenneté dans la Nouvelle-Zélande contemporaine au 
regard de la biculturalité de son histoire. Le même biculturalisme donne l'intitulé de l'exposé de Kelly Barclay qui propose de "repenser l'inclusion et le biculturalisme " (pp.118-139) à la lumière d'une "pratique plus relationnelle de la justice démocratique ", entendant par là une justice qui tienne compte d'un point de vue non occidental dans ses attendus et ses jugements.

9 En regardant «le visage changeant de la population néo-zélandaise » (pp.140-154) Arvind Zodgekar dresse le tableau des provenances des flux migratoires sur 30 ans et constate un accroissement en pourcentage de la population maori (et parallèlement de la population extra-occidentale), en même temps qu'une diminution concomitante de la population occidentale. L'entrée en lice des populations insulaires du Pacifique et de populations d'origine asiatique est manifeste sur la période considérée.

10 Colleen Ward et En-Yi Lin essaient de bâtir la corrélation entre «immigration, acculturation et identité nationale en Nouvelle-Zélande » (pp. 155-173) en se fondant sur les chiffres des stratégies exprimées en termes d'intégration, d'assimilation, de séparation et de marginalisation. À $80 \%$ sur un effectif de 772 adolescents interrogés, la préférence va à la stratégie d'intégration, mais la tendance à la marginalisation n'est pas exclue chez les populations originaires des îles du Pacifique.

11 L'identité néo-zéalandaise revendiquée par les Chinois installés dans le pays fait l'objet d'une étude spécifique de Manying Ip et David Pang (pp.174-190) et ses résultats diffèrent suivant qu'il s'agit de résidents, de nationaux ou de travailleurs temporaires. Les liens avec les mères-patries restent fortement attestés.

12 De son côté, Belinda Borell étudie la notion d'identité culturelle chez les jeunes Maori de la banlieue sud d'Auckland (pp. 191-206) et en tire la conclusion que la situation n'est pas jugée «au plus mal » par les premiers concernés, malgré les désavantages matériels et les gangs, en raison de l'environnement majoritairement maori estimé favorable au développement local. Teresia Teaiwa et Sean Mallon s'intéressent pour leur part aux populations originaires du Pacifique (pp. 207-229), en particulier des îles Samoa, Tokelau et Cook, anciennement sous mandat néo-zélandais, ce qui leur confère une sorte de " double parenté » nationale. L'analyse porte en particulier sur l'entrée de ces populations sur les scènes du rugby et des médias (théâtre et télévision) en montrant qu'il s'agit d'une entrée certes minoritaire mais significative en termes d'image nationale multiculturelle.

13 La représentation de la Nouvelle-Zélande à l'extérieur, notamment au niveau de sa diplomatie, a retenu l'attention de David Capie et Gerald McGhie (pp. 230-241). Ces auteurs conviennent que la Nouvelle-Zélande a progressivement acquis une «identité du Pacifique ", jouant un rôle prépondérant avec l'Australie sur le plan régional des États insulaires.

14 «Qui sommes-nous? » est la question que traite Paul Morris (pp. 242-254) en abordant l'incidence de l'appartenance religieuse sur la conscience individuelle et collective en Nouvelle-Zélande. Les appartenances religieuses s'étant considérablement diversifiées dans les dernières décennies et les «mouvements prophétiques maori » n'étant plus jugés comme marginaux, la question d'une spiritualité commune dans l'ensemble national constitué ne va pas de soi, et les positions intégratives adoptées par certains pays européens face à l'islam par exemple ressurgissent collatéralement en NouvelleZélande en réinterrogeant la spiritualité à l'américaine, faite d'un consensus autour des valeurs chrétiennes fortement proclamées. 
15 Une table ronde affichant modestement dans son titre «100\% de conjecture » pour bâtir des scénarios pour l'avenir de la Nouvelle-Zélande (pp. 255-290) propose, à l'aide de photographies anciennes de populations européennes implantées dans ce pays, trois actes virtuels d'évolution des enjeux citoyens sur les deux décennies à venir. Dans une postface, Joris de Bres, commissaire aux relations raciales (pp. 291-292) conclut sur l'obligation d'une identité nationale « inclusive » pour l'avenir de son pays. Ce livre d'actes est plus qu'un livre dédié à la seule Nouvelle-Zélande. Loin de se complaire dans une situation qui serait isolée et isolationniste, les auteurs se réfèrent constamment à la situation d'autres pays du monde, notamment occidentaux. On dispose ainsi d'un beau programme d'analyse scientifique des questions identitaires qui mériterait à plus d'un titre d'être relayé et appliqué dans des pays réputés de «vieille souche ». 